\title{
Cathodoluminescence studies of growth and process-induced defects in bulk gallium antimonide
}

\author{
B. Méndez \\ Departamento de Fisica de Materiales, Facultad de Fisicas, Universidad Complutense, \\ E-28040 Madrid, Spain \\ P. S. Dutta \\ Department of Physics, Indian Institute of Science, Bangalore-560 012, India \\ J. Piqueras \\ Departamento de Fisica de Materiales, Facultad de Fisicas, Universidad Complutense, \\ E-28040 Madrid, Spain \\ E. Dieguez \\ Departamento de Fisica de Materiales, C-IV, Universidad Autonoma, Madrid-28049, Spain
}

(Received 27 April 1995; accepted for publication 22 August 1995)

\begin{abstract}
The homogeneity and luminescence properties of undoped bulk GaSb have been studied by the cathodoluminescence $(\mathrm{CL})$ technique in the scanning electron microscope. CL images have revealed a nonuniform distribution of native defects in GaSb wafers prepared from as-grown single crystals. Postgrowth annealing in vacuum, gallium, and antimony atmospheres has been performed to obtain more accurate information about the defect structure in this material. In general, on annealing, homogeneous distribution of impurities is observed throughout the wafers. CL spectra show that a luminescence band (centered at $756 \mathrm{meV}$ ) is enhanced by annealing in a gallium atmosphere, suggesting that $\mathrm{Ga}$ atoms play an important role in the formation of this acceptor center. The 756 meV peak has been attributed to a transition from conduction band to an acceptor center comprised of $\mathrm{Ga}_{\mathrm{Sb}}$ or a related complex. Interestingly, localized crystallization at the subgrain boundaries seems to occur by annealing in Ga atmosphere. () 1995 American Institute of Physics.
\end{abstract}

Recently, $\mathrm{ZrF}_{4}$-based fluoride glass optical fibers have been predicted to have intrinsic minimum losses one or two orders of magnitude lower than those of conventional silica fibers. ${ }^{1}$ The loss minima for the next generation fibers are expected to occur in the 2-4 $\mu \mathrm{m}$ wavelength regime. These estimations have stimulated considerable interest on materials for mid-IR sources and detectors. GaSb is the most suitable substrate material for various lattice-matched optoelectronic devices in the range of $0.3 \mathrm{eV}$ (InGaAsSb) $-1.58 \mathrm{eV}$ $(\mathrm{AlGaAsSb}) .^{2-4}$ There are several reports on the growth of bulk GaSb single crystals employing various techniques. ${ }^{5-7}$ However, there are very few reports on the characterization of defects in the grown crystals. For defect characterization, the cathodoluminescence (CL) technique has been found to be highly sensitive and is extensively used for the spatial defect mapping. To the best of our knowledge, CL microscopy has not been applied to defects studied in GaSb. In this letter, we present the results of CL investigation of defects in bulk GaSb generated during growth and postgrowth annealing treatments.

The GaSb samples used in our studies were vertical Bridgman grown single crystals. ${ }^{8}$ Undoped GaSb is always $p$ type in nature with the acceptor concentration of approximately $10^{17} \mathrm{~cm}^{-3}$ at room temperature. The acceptor is intrinsic and is due to a vacant gallium site $\left(V_{\mathrm{Ga}}\right)$ and gallium antisite $\left(\mathrm{Ga}_{\mathrm{Sb}}\right)$. The acceptor concentration can be reduced either by nonstoichiometric melt growth or by employing low-temperature growth techniques. ${ }^{9,10}$ In this work we have performed postgrowth annealing in vacuum, gallium, and antimony atmospheres to examine the evolution and nature of the native acceptors. The wafers were prepared by conventional chemomechanical polishing. Prior to annealing, the samples were etched in $\mathrm{CH}_{3} \mathrm{COOH}: \mathrm{HNO}_{3}: \mathrm{HF}$ (20:9:1) to remove any damaged layer left behind after polishing, dipped in $\mathrm{HCl}$, and rinsed in methanol. Thermal annealing of the samples was carried out by placing the wafers in a quartz ampoule under vacuum. For the gallium annealing, $6 \mathrm{~N}$ pure gallium was spread on the wafer surface at room temperature. After annealing, the gallium sticking to the surface was removed by rinsing in $\mathrm{HCl}$. For annealing in antimony atmosphere, $6 \mathrm{~N}$ pure antimony balls were kept along with the wafer. Other wafers were annealed under a vacuum of $10^{-6}$ Torr. The annealing temperature and time were kept constant at $500{ }^{\circ} \mathrm{C}$ and $12 \mathrm{~h}$, respectively, for all the cases. The CL measurements were carried out using a Hitachi S-2500 scanning electron microscope in the emissive and CL modes at $77 \mathrm{k}$. A liquid-nitrogen-cooled North Coast EO-817 germanium detector was used for the signal detection. The details of the experimental setup for spectral and panchromatic CL measurements are presented elsewhere. ${ }^{11}$

The broad CL spectra of various samples have been deconvoluted using the Gaussian functions with well-known luminescence peak positions in GaSb. From the best fits, the presence of various peaks has been inferred. Figure 1 shows the CL spectrum of the as-grown GaSb sample. The dominant emission bands are at 796 and $775 \mathrm{meV}$. Weak transitions at 756 and $830 \mathrm{meV}$ can also be seen. The $796 \mathrm{meV}$ corresponds to the band-band transition and the $775 \mathrm{meV}$ (commonly known as band $A$ ) to a transition from the conduction band to the neutral state of the native acceptor level 


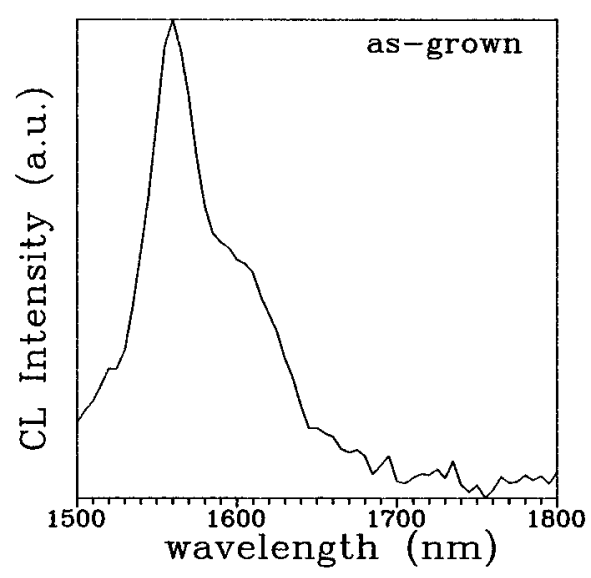

FIG. 1. CL spectrum at $77 \mathrm{~K}$ of an as-grown undoped GaSb sample.

$\mathrm{V}_{\mathrm{Ga}} \mathrm{Ga}_{\mathrm{Sb}} \cdot{ }^{12-14}$ The peak at $756 \mathrm{meV}$ (designated as band $B$ ) has been previously attributed to a transition from conduction band to a triple native acceptor complex $\mathrm{V}_{\mathrm{Ga}} \mathrm{Ga}_{\mathrm{Sb}} \mathrm{V}_{\mathrm{Ga}}$, arising from an excess of Ga vacancies. ${ }^{15}$ However, the origin of this transition has not been clearly resolved until now. ${ }^{16}$ The above-band-gap peak at $830 \mathrm{meV}$ is an $(e, h)$ transition including tail states and shallow acceptors ${ }^{17}$ and is observed here due to high excitation intensities in CL.

On annealing under different ambients, the band-band
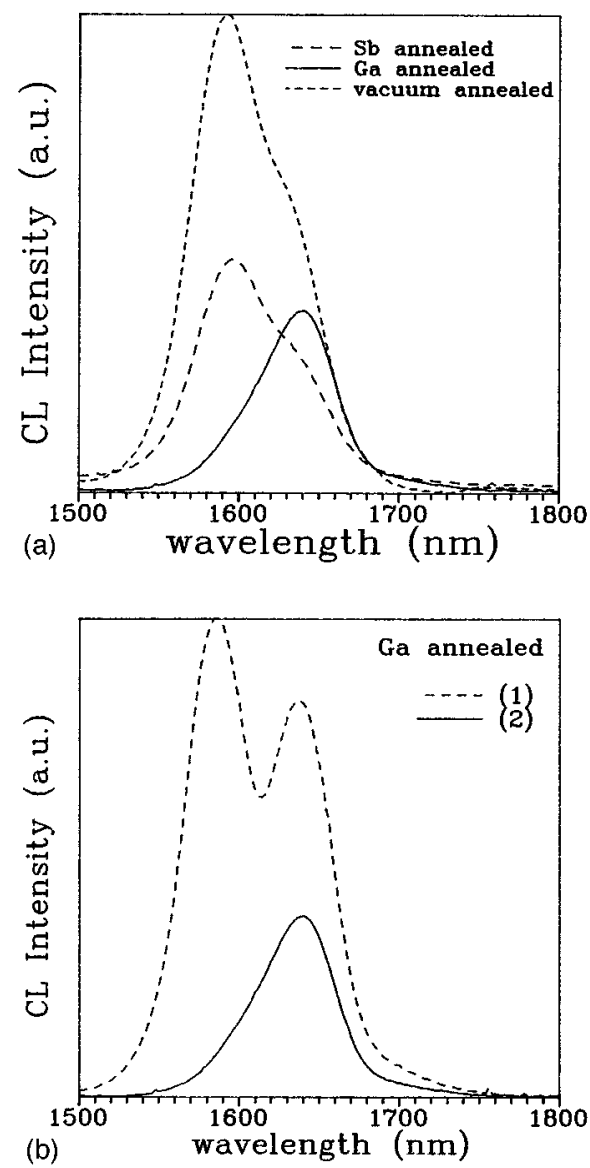

FIG. 2. CL spectra at $77 \mathrm{~K}$ of (a) vacuum-, antimony-, and gallium-annealed $\mathrm{GaSb}$ at $500^{\circ} \mathrm{C}, 12 \mathrm{~h}$, and (b) CL spectra from different positions of the gallium-annealed sample.
(796 meV) and the above-band-gap (830 meV) transitions totally disappear [see Fig. 2(a)]. This implies that the 796 $\mathrm{meV}$ band may be due to a transition from the indirect free electron to the valence band tails, created by the inhomogeneous impurity distribution. It has been confirmed from CL imaging that annealing leads to homogenization of defects. Therefore, the $796 \mathrm{meV}$ transition is no longer seen in the annealed samples. Apart from the homogeneity effect, some changes in the nature of luminescence bands are also observed. The annealed samples exhibit only the 775 and 756 $\mathrm{meV}$ transitions, but with a difference in relative intensities depending on the annealing conditions. In general, the relative intensity of the $775 \mathrm{meV}$ with respect to the $756 \mathrm{meV}$ transition decreases after annealing. This can be either due to the decrease in $775 \mathrm{meV}$ or increase in $756 \mathrm{meV}$ peak intensity. The vacuum and Sb-annealed samples show a similar CL spectrum. Moreover, the relative intensity of the 775 $\mathrm{meV}$ with respect to the $756 \mathrm{meV}$ transition is less in the Sb-rich samples. From the CL spectrum of the Ga-annealed sample, one can infer about the possible origin of the 756 $\mathrm{meV}$ transition. In the Ga-annealed sample, the $756 \mathrm{meV}$ transitions enhance drastically and at certain locations of the sample, it is even more than the $775 \mathrm{meV}$ peak as shown in Fig. 2(b). Thus the band $B$ transition is associated with a defect involving excess gallium and can be due to a gallium antisite or a related complex. Hence the decrease in relative intensity of the $775 \mathrm{meV}$ peak compared to the $756 \mathrm{meV}$ in the Sb- and vacuum-annealed samples can be explained by considering the reduction of the native acceptors on annealing, which shifts the Fermi level position towards the conduction band and hence the intensity of the $756 \mathrm{meV}$ transition (which is weakly present in the as-grown sample) increases. Also, the native defect concentration is less in Sbannealed samples than in the vacuum-annealed ones. On the other hand, gallium atoms enhance the formation of the centers responsible for the band $B$ emission at the same time that induces the rupture of the native defect associated with band $A$. Further, it has been observed that the absolute CL intensity also decreases after annealing in a $\mathrm{Sb}$ atmosphere. This

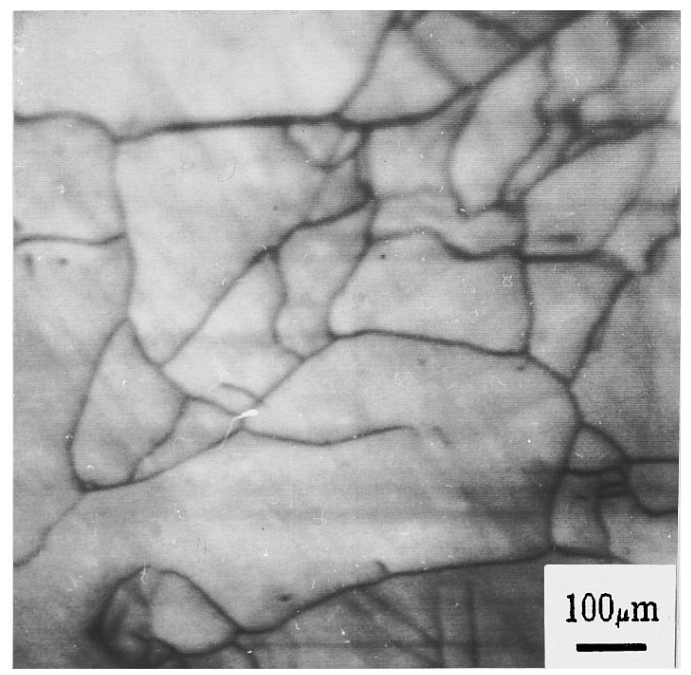

FIG. 3. CL image of as-grown undoped GaSb. 


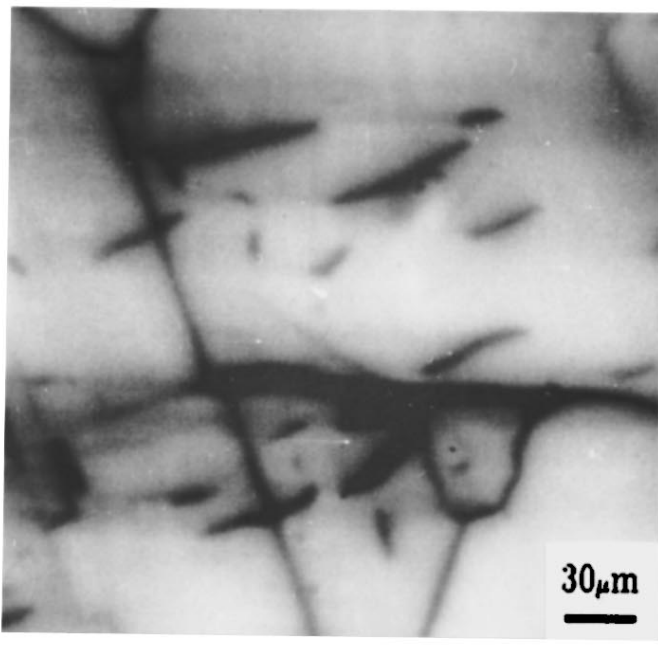

(a)

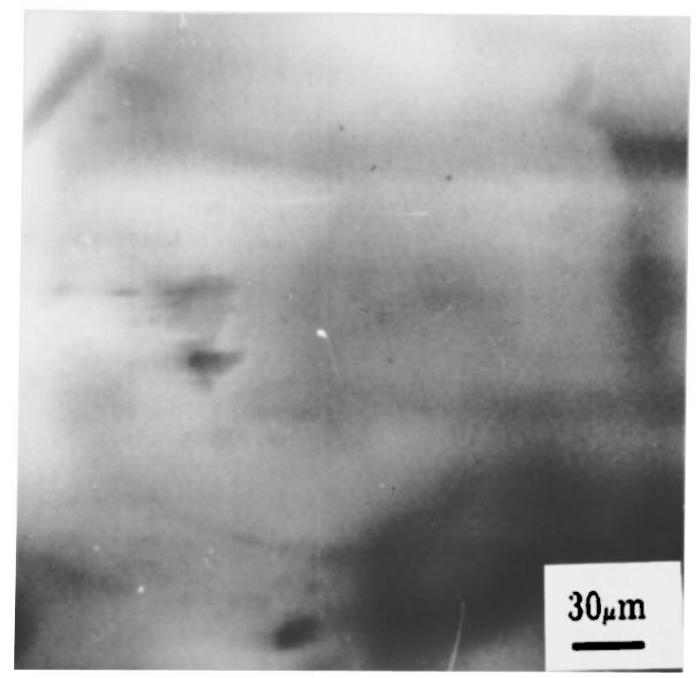

(b)

FIG. 4. CL images of (a) antimony- and (b) gallium-annealed samples.

can be due to the generation of nonradiative recombination centers. To explore such a possibility and achieve a better understanding of the defect centers, we have complemented the spectral study with CL images recorded at various locations on all the samples.

The CL image of as-grown GaSb shows a dark contrast related to the presence of a subboundary in the material (Fig. 3 ). The inner portion of the grains appears uniformly bright. The CL images of the Sb-annealed sample revealed dark precipitatelike defects which contribute to the reduction of luminescence emission [Fig. 4(a)]. These defects are not seen in as-grown or vacuum-annealed samples. The defect structure in the vacuum-annealed sample is similar to that of the untreated ones. The Ga-annealed sample exhibits a uniform CL image [see. Fig. 4(b)]. Taking into account that the emission is dominated by band $B$, this observation suggests that the associated centers are uniformly distributed in the annealed wafer. The possible origin of contrast in the as-grown samples is due to the presence of excess interstitial Sb which occupies the subboundaries after volatilization during the growth of crystals. The vacant $\mathrm{Sb}$ site in the bulk would then lead to the formation of the doubly ionizable native defect complex $\mathrm{V}_{\mathrm{Ga}} \mathrm{Ga}_{\mathrm{Sb}}$. By annealing in a Ga solution, localized crystallization at the subboundaries can take place to form GaSb. This would eliminate the excess $\mathrm{Sb}$ at the subboundaries and the defect centers are uniformly generated. The process is similar to the growth of $\mathrm{GaSb}$ from liquid phase at low temperature from a Ga-rich melt. After annealing, the sample exhibits uniform defect distribution with no contrast. Such a localized crystallization process is not possible during annealing in vacuum or $\mathrm{Sb}$ ambients and hence the CL images are similar to that of the as-grown sample. Thus postgrowth annealing in $\mathrm{Ga}$ bath can be technologically important for preparing GaSb wafers with uniform properties for optoelectronic applications.

In conclusion, we have performed CL measurements on bulk GaSb wafers. Nonuniform defect distribution is revealed in the as-grown undoped samples. After annealing in vacuum, Ga- and Sb-atmosphere homogenization of defects in the bulk occurs. Even though annealing in Sb atmosphere reduces the native defect concentration, precipitate formation takes place due to which the overall luminescence intensity reduces. On the other hand, Ga-annealed samples exhibit featureless CL images suggesting the highly uniform nature of the defect distribution. Furthermore, it is proved that the 756 $\mathrm{meV}$ transition (band $B$ ) is related to excess $\mathrm{Ga}$ in the sample. For large scale technological applications, annealing of as-grown $\mathrm{GaSb}$ wafers in Ga bath can be employed for the production of high quality device grade substrates.

This work has been supported by the DGICYT (Project No. PB93-1256) and ESP95-0148. One of the authors (P.S.D.) gratefully thanks CSIR (India) for the award of the Senior Research Fellowship and UAM, Madrid, Spain, for the visiting scientist fellowship.

${ }^{1}$ M. G. Drexhage and C. T. Moyniham, Sci. Am. 259, 76 (1988).

${ }^{2}$ A. G. Milnes and A. Y. Polyakov, Solid State Electron. 36, 803 (1993).

${ }^{3}$ A. Sasaki, M. Nishiuma, and Y. Takeda, Jpn. J. Appl. Phys. 19, 1695 (1980).

${ }^{4}$ M. J. Cherng, G. B. Stringfellow, D. W. Kisker, A. K. Srivastava, and J. L. Zyskind, Appl. Phys. Lett. 48, 419 (1986).

${ }^{5}$ C. Woelk and K. W. Benz, J. Cryst. Growth 27, 177 (1977).

${ }^{6}$ E. T. R. Chidley, S. K. Haywood, A. B. Henriques, N. J. Mason, R. J. Nicholas, and P. J. Walker, Semicond. Sci. Technol. 6, 45 (1991).

${ }^{7}$ T. M. Rossi, D. A. Collins, D. H. Chow, and T. C. McGill, Appl. Phys. Lett. 57, 2256 (1990).

${ }^{8}$ P. S. Dutta, K. S. Sangunni, H. L. Bhat, and V. Kumar, J. Cryst. Growth 141, 44 (1994).

${ }^{9}$ C. Anayama, T. Tanahashi, H. Kuwatsuka, S. Nishiyama, S. Isozumi, and K. Nakajima, Appl. Phys. Lett. 56, 239 (1990).

${ }^{10}$ M. C. Wu, C. W. Chen, and C. C. Chen, J. Appl. Phys. 72, 1101 (1992).

${ }^{11}$ B. Méndez and J. Piqueras, J. Appl. Phys. 69, 2776 (1991).

${ }^{12}$ W. Jakowetz, W. Ruhle, K. Breuninger, and M. Pilkuhn, Phys. Status Solidi A 12, 169 (1972)

${ }^{13}$ M. Lee, D. J. Nicholas, K. E. Singer, and B. Hamilton, J. Appl. Phys. 59, 2895 (1986).

${ }^{14}$ M. C. Wu and C. C. Chen, J. Appl. Phys. 72, 4275 (1992).

${ }^{15}$ D. Weiler and H. Mehrer, Philos. Mag. A 49, 309 (1984).

${ }^{16}$ W. J. Jiang, Y. M. Sun, and M. C. Wu, J. Appl. Phys. 77, 1725 (1995).

${ }^{17}$ G. Benz and R. Conradt, Phys. Rev. B 16, 843 (1977). 